\title{
Functional renormalization group for the anisotropic triangular antiferromagnet
}

\author{
Johannes Reuther ${ }^{1}$ and Ronny Thomale $^{2}$ \\ ${ }^{1}$ Institut für Theorie der Kondensierten Materie, \\ Karlsruhe Institute of Technology, 76128 Karlsruhe, Germany \\ ${ }^{2}$ Department of Physics, Princeton University, Princeton, NJ 08544, USA
}

(Dated: August 14, 2018)

\begin{abstract}
We present a functional renormalization group scheme that allows us to calculate frustrated magnetic systems of arbitrary lattice geometry beyond $\mathcal{O}(200)$ sites from first principles. We study the magnetic susceptibility of the antiferromagnetic (AFM) spin-1/2 Heisenberg model ground state on the spatially anisotropic triangular lattice, where $J^{\prime}$ denotes the coupling strength of the intrachain bonds along one lattice direction and $J$ the coupling strength of the interchain bonds. We identify three distinct phases of the Heisenberg model. Increasing $\xi=J^{\prime} / J$ from the effective square lattice $\xi=0$, we find an AFM Néel order to spiral order transition at $\xi_{c 1} \sim 0.6-0.7$, with indication to be of second order. In addition, above the isotropic point at $\xi_{c 2} \sim 1.1$, we find a first order transition to a magnetically disordered phase with collinear AFM stripe fluctuations.

PACS numbers: $75.10 . \mathrm{Jm}$
\end{abstract}

Frustration induced by lattice geometry and interaction in two-dimensional quantum antiferromagnets constitutes a considerable challenge beyond mean field theory [1]. This is the main reason why the question of distinguishing systems with magnetic long-range order and spin liquid behavior has been one of the most difficult and long-standing problems in the field of quantum magnetism. A plethora of different methods has been developed to address this problem in the context of many different lattices and magnetic Hamiltonians, most of which, however, have severe drawbacks of very different kind. Quantum Monte Carlo (QMC) methods [2] may suffer from the sign problem often encountered for frustrated systems, while exact diagonalization (ED) studies are constrained to small system sizes. Density matrix renormalization group (DMRG) methods [3, 4] partly resolve the latter problem, but are constrained to (effectively) one-dimensional systems. Coupled cluster methods (CCM) [5] and variational QMC methods [ [6] are valuable approaches to treat frustrated two-dimensional systems. However, they are limited in the sense that, generally, only trial state energies can be tested against each other, and no unbiased treatment from first principles is possible.

Promising lines of improvement have been undertaken recently. In special cases where it is applicable, dimer projection schemes allow to treat larger systems at a similar level of precision and completeness as ED [7]. From a perturbative expansion perspective, continuous unitary transformation (CUT) methods provide a helpful tool to compute the energies of the ground state and excitation modes of small quasiparticle sectors [8, 9]. Furthermore, the generalized notion of matrix product states, in certain cases, leads to an efficient treatment of two-dimensional magnetic systems by projected entangled pair states (PEPS) [10]. In particular, the multiscale entanglement renormalization Ansatz (MERA) pro- vides a new tool to compute energies and scaling behavior of certain magnetic systems, and has been most recently applied to frustrated systems 11, 12]. Still, even in the optimal cases where the approximations made within these methods are controlled, it is mainly suited to determine certain ground state properties only. For frustrated magnetic systems and actual comparison to experiment, however, it would be most desirable to compute complementary thermodynamic quantities that allow to resolve the competition and classification of magnetic ordering and quantum fluctuations. The most suitable quantity in this respect is the magnetic susceptibility. Interpreted as the magnon spectral function, it provides detailed information about the qualitative and quantitative type of magnetic fluctuations, and is the canonic quantity measured in experiment. In this Letter, we employ the pseudofermion functional renormalization group (PFFRG) 13] as a new method to tackle systems of frustrated magnetism. Applying the method to the anisotropic triangular lattice, we demonstrate that the PFFRG is able to (i) treat large system sizes of $\mathcal{O}(200)$ sites, (ii) is applicable to arbitrary frustrated lattice geometries and two-body bare interactions, (iii) naturally allows to compute the magnetic susceptibility as the canonical outcome of the RG, and (iv) hence provides an unbiased calculation from first principles that allows comparison to experiment.

The Heisenberg model on the anisotropic triangular lattice (ATLHM) attracted considerable attention in recent years as an experimentally accessible testing ground for quantum magnetism disorder phenomena. The Hamiltonian is given as

$$
H_{\mathrm{ATLHM}}=J \sum_{\langle i, j\rangle_{\mathrm{v}}} \vec{S}_{i} \vec{S}_{j}+J^{\prime} \sum_{\langle i, j\rangle_{-}} \vec{S}_{i} \vec{S}_{j}
$$

where the coupling $J^{\prime}$ applies to the bonds along (horizontal) one-dimensional chains and $J$ is the coupling between them, forming a triangular lattice altogether 

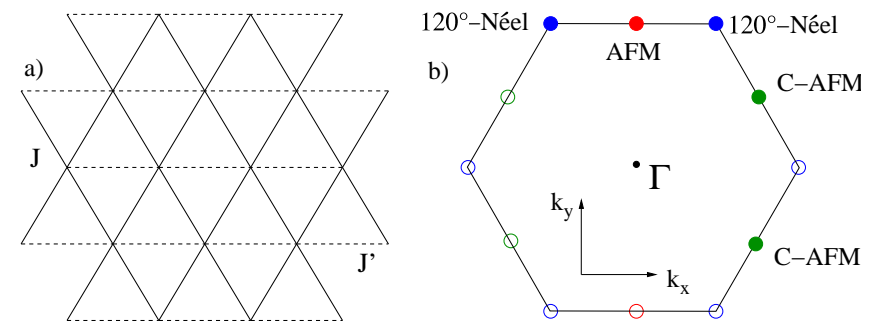

FIG. 1. (color online) a) Triangular lattice structure. The horizontal bonds correspond to coupling $J^{\prime}$ the other to coupling $J$. b) Schematic plot of the hexagonal Brillouin zone. Different magnetic order resides at different points (shown: AFM Néel order, $120^{\circ}$ Néel order, collinear AFM order (cAFM)). The open circles relate to the filled circles by reciprocal lattice vectors, i.e., AFM order corresponds to two points in the BZ, collinear AFM to four points, and $120^{\circ}$ Néel order to six points.

(Fig. 1h). We define $\xi=J^{\prime} / J$ as a parameter to interpolate between the effective square lattice limit $\xi=0$ and the disordered isolated chain limit $\xi \rightarrow \infty$. Experiments on $\mathrm{Cs}_{2} \mathrm{CuCl}_{4}$ 14] $(\xi \sim 2.94)$ provide a good testing ground of discussing various features of spin liquid behavior. The formation of a magnetically ordered state for smaller $\xi$ opposed to disorder tendencies for larger $\xi$ can be nicely studied for the organic $\kappa-(\mathrm{BEDT}-\mathrm{TTF})_{2} \mathrm{X}$ family. While $\mathrm{X}=\mathrm{Cu}_{2}\left[\mathrm{~N}(\mathrm{CN})_{2}\right] \mathrm{Cl}$ shows an AFM transition of $T_{\mathrm{N}}=27 \mathrm{~K}$ with estimated $\xi=0.55$, the $\mathrm{X}=\mathrm{Cu}_{2}(\mathrm{CN})_{3}$ compound, estimated to be nearby the symmetric triangular regime $\xi=1.15$, does not show magnetic order down to very low temperatures [15]. Details of the phase diagram of (10) are still of current debate. It is an established fact that the system is AFM Néel-ordered for small $\xi$, changing to $120^{\circ}$ Néel order as the isotropic triangular limit $\xi=1$ is reached [17 23]. However, an intermediate disordered phase has been proposed 24], while other works assume a direct transition, but cannot classify the transition to be of second or first order [21, 22]. For larger $\xi>1$, some works claim a disordered phase extending to the $\xi \rightarrow \infty$ limit [19, 25], whereas others indicate collinear antiferromagnetic (cAFM) ordering [22, 23, 26].

We now address this problem with PFFRG, which is explained in more detail in [13]. Unlike conventional FRG studies, our starting point is not given by the bare excitations of the spin system. Instead, we use the pseudofermion representation of spin- $1 / 2$ operators $S^{\mu}=1 / 2 \sum_{\alpha \beta} f_{\alpha}^{\dagger} \sigma_{\alpha \beta}^{\mu} f_{\beta},(\alpha, \beta=\uparrow, \downarrow, \mu=x, y, z)$ with the fermionic operators $f_{\uparrow}$ and $f_{\downarrow}$ and the Pauli-matrices $\sigma^{\mu}$. This representation enables us to apply Wick's theorem leading to standard Feynman many-body techniques. The introduction of pseudofermions comes along with an artificial enlargement of the Hilbert space and therefore requires the fulfillment of an occupancy constraint (exclusion of empty and doubly occupied states). Since an unphysical occupation acts as a vacancy in the spin lattice (a)

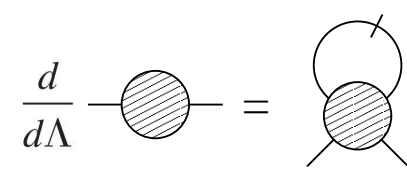

(b)

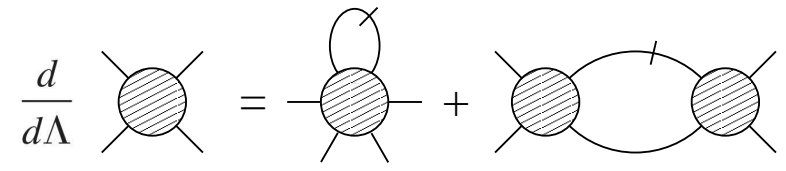

(c)

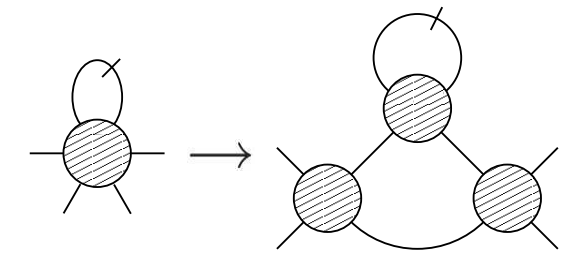

(d)

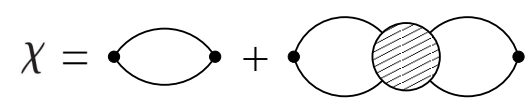

FIG. 2. Graphic representation of the FRG differential equations. Bare lines denote the (renormalized and scale dependent) Green's functions and slashed lines the single scale propagators. Eqs. (a) and (b) are the FRG equations for the self-energy and the two-particle vertex, respectively (without distinguishing between the different pairing channels on the right side of (b)). The Katanin scheme is given by the replacement (c). Fusing the external legs of the two-particle vertex, the magnetic susceptibility is obtained as shown in (d).

associated with an excitation energy of order $J$, particle number fluctuations are suppressed at zero temperature and the constraint is naturally fulfilled. Quantum spin models are inherently strong coupling models, requiring infinite self-consistent resummations of perturbation theory. In this context FRG 27 30] provides a systematic summation in different interaction channels by generating equations for the evolution of all one-particle irreducible $m$-particle vertex functions under the flow of an IR frequency cutoff $\Lambda$ (see Fig. $2 \mathrm{a}$ and $2 \mathrm{~b}$ for the flow of the self-energy and the two-particle vertex). In order to reduce the infinite hierarchy of equations to a closed set, some truncation scheme has to be applied. As an important difference in the PFFRG as compared to conventional FRG schemes, we still include certain 2-loop contributions that are shown by Katanin [31] to be essential for a better fulfillment of Ward-identities (see Fig. 2. In particular, this way the random phase approximation (RPA) is recovered as a diagram subset generated by the RG flow [32]. It is important to emphasize that the RPA diagrams play a crucial role in our scheme as they are responsible for the collective ordering phenomena. In addition, however, our method treats the leading diagrammatic contributions of a $1 / S$ expansion 35, 36] (direct particle-hole graphs) , 1/N expansion 33, 34] (crossed particle-hole graphs), and particle-particle diagrams on the same footing. This enables us to adequately ac- 

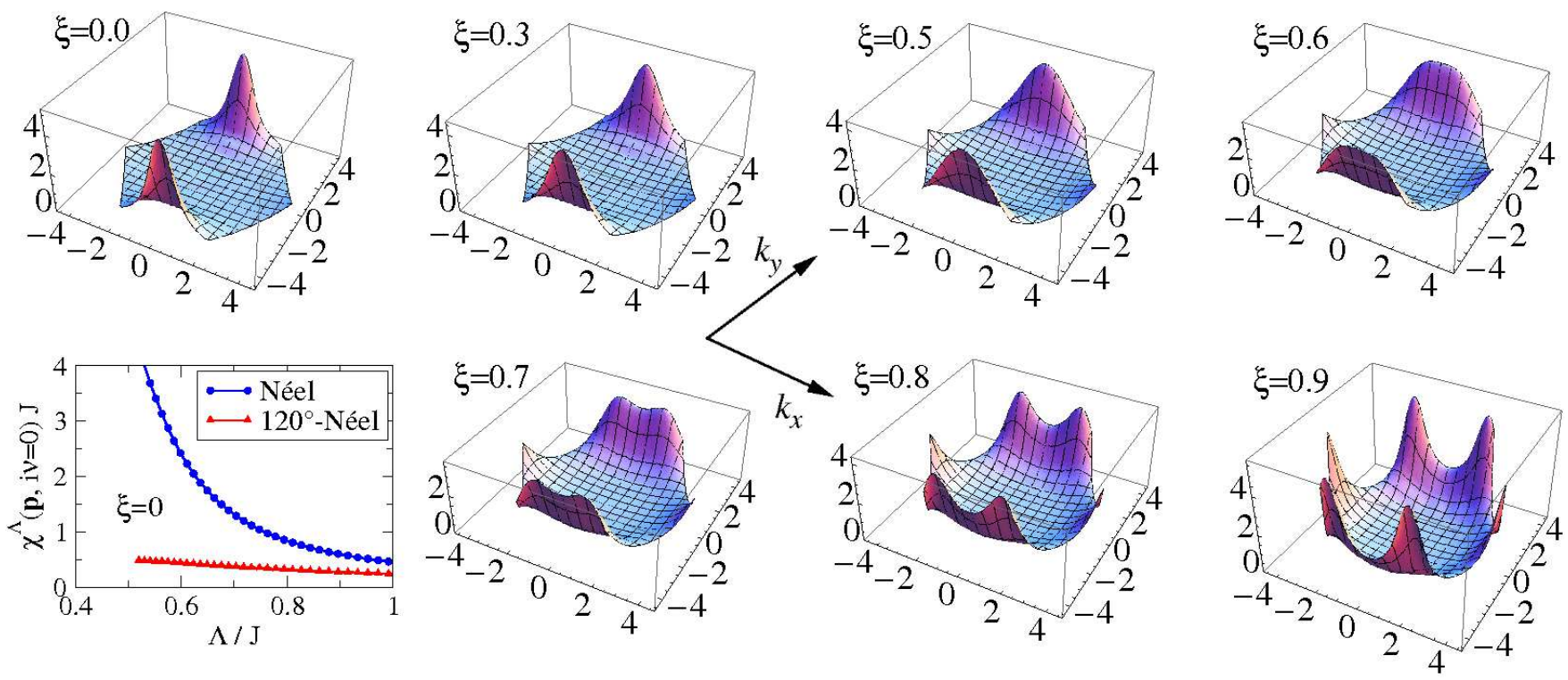

FIG. 3. (color online) Static magnetic susceptibility resolved for the whole Brillouin zone, varying $\xi=J^{\prime} / J$ from the effective square lattice $\xi=0.0$ close to the triangular lattice $\xi=0.9$. Bottom Left: 2-particle vertex flow for the AFM Néel channel (blue) versus the $120^{\circ}$ Néel channel (red) at $\xi=0$ with respect to the IR cutoff flow parameter $\Lambda$. We observe that the AFM vertices start to diverge, signalling a magnetic instability. Susceptibilities are always given in units of $1 / J$. The respective types of order for the distinct peaks positions are shown in Fig. 1 1 b.

count for the competition of magnetic order and disorder in an unbiased fashion. The magnetic susceptibility can be conveniently computed from the two-particle vertex (Fig. 2 $\mathrm{d}$ ). Due to the local nature of the auxiliary fermions of a bare spin model, a real-space representation of all vertices is much more suitable than the usual momentum space scheme. Furthermore, since we operate in the strong coupling limit, the proper regularization of Green's functions requires the self-consistent back coupling of the self energy (Fig. 2 2 ) into the propagators in Fig. 2 2 . In order to account for the dynamic fluctuations, it is also necessary to keep all frequency dependencies of the vertex functions involved. The numerical solution requires the discretization of these frequencies. Similarly, the spatial dependence is approximated by keeping correlation functions of an infinite system up to a maximal length. In our calculations, this length extends over 7 lattice spacings, leading to a correlation area of 169 lattice sites (this constrains the resolvable incommensurable order to vector sizes within that range). An ordering instability is initially signalled by a strong rise of the vertex couplings associated with this order at some finite scale of $\Lambda$ (Fig. 3 and 4). Since in the present formulation rotational invariance is conserved during the flow, we should not find stable solutions down to $\Lambda=0$ in magnetically ordered regimes. Indeed, the onset of spontaneous long range order is signalled by a sudden breakdown of the smooth flow. In contrast, the existence of a stable solution indicates the absence of long range order associated with spin rotational symmetry breaking. In the present implementation, our approach does not directly detect chiral order. This is because the chirality operator is a three-body operator by construction, and hence relates to the 3-particle vertex whose explicit RG flow is not computed.

We sweep through the parameter space of $\xi$ from the square lattice to the isotropic triangular lattice and compute the static magnetic susceptibility shown in Fig. 3 (the peak positions for different types of long range order are depicted in Fig. 1b). Throughout this parameter regime, we observe a characteristic breakdown of the flow, indicating ordering instabilities rather than a disordered phase. One can nicely observe how the susceptibility evolves as we increase $\xi$. As shown in Fig. 3. we find a broadening and subsequent splitting of the Néel-peak. The splitted peaks evolves along the Brillouin-zone edge and the weight at the corners of the hexagon increases until in the isotropic case the peak symmetrically resides at the corner positions. From the splitting of the Néel peak, we estimate the transition to be at $\xi_{c 1} \sim 0.6-0.7$, i.e., the regime above which the order becomes incommensurate. Apparently, the system influenced by quantum fluctuation favors AFM fluctuations over spiral fluctuations, since the classical transition point at $\xi=0.5$ is shifted to higher $\xi$. In particular, from the extremely smooth evolution of the susceptibility upon variation of $\xi$ which we studied by steps of 0.02 in $\xi$, we find indication for the transition to be of second order, while an extremely weak first order transition (corresponding to a slight kink in the leading susceptibility channel) cannot be excluded as a matter of principle. The height of the peaks such as in Fig. 3 still depend on system size. 

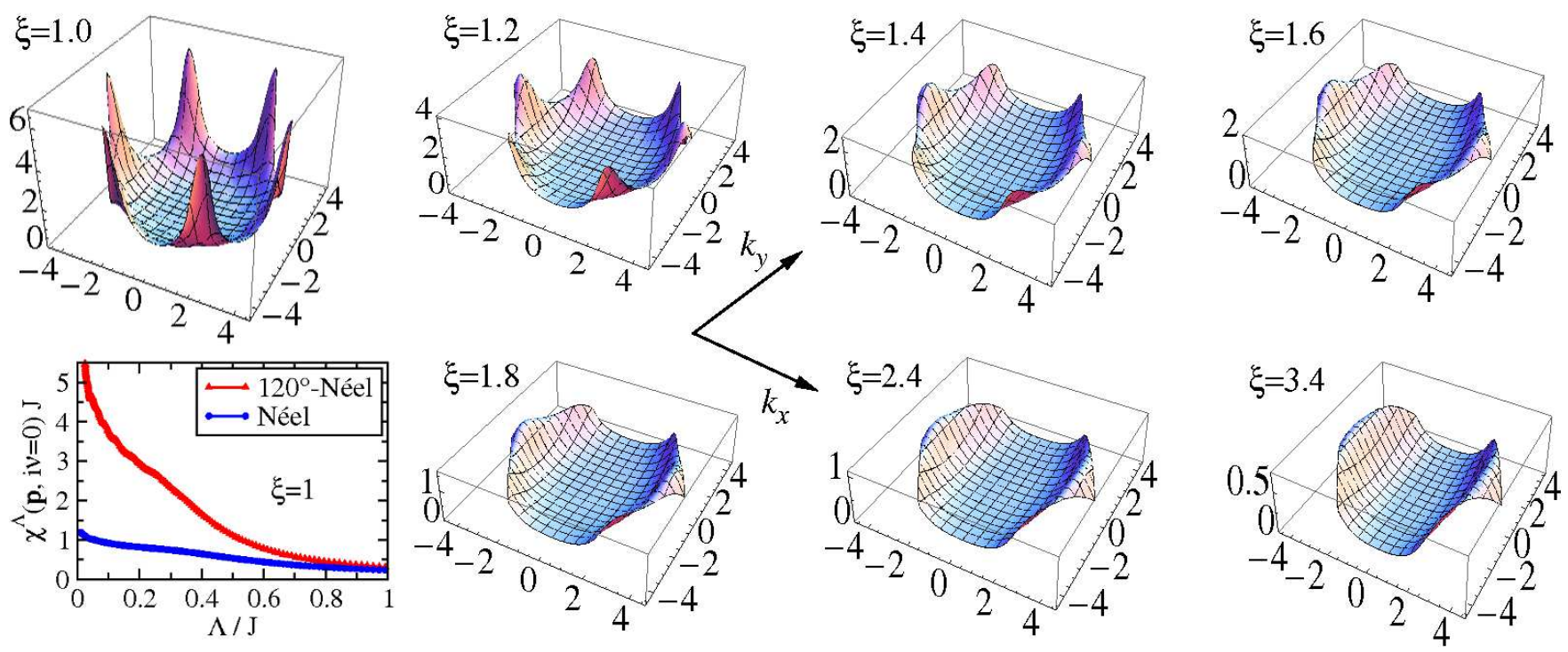

FIG. 4. (color online) Static magnetic susceptibility, varying $\xi$ from the isotropic triangular lattice $\xi=1.0$ towards the $1 \mathrm{~d}$ chain limit $\xi \rightarrow \infty$. Bottom left: Vertex flow at the $\xi=1$ point. The rise of the $120^{\circ}$ Néel channel shows the ordering instability at the isotropic triangle point. Compared to the flow at $\xi=0$ (Fig. 3), the rise takes place at a much lower scale of $\Lambda$, indicating a lower ordering scale for the triangular lattice.

At the isotropic triangular point $\xi=1$ where additional lattice symmetries enable us to consider systems even beyond 250 sites, we obtain higher and sharper peaks with increasing system size.

In a recent work, Starykh and Balents have found that for $\xi>1$, the quantum system enters a collinear antiferromagnetic stripe phase 26]. This is interesting since the classical estimate would be spiral order in that regime, so the quantum fluctuations lead to a different ordering [23]. However, finite size numerical studies reported a disordered phase in that regime 21]. Our results for the magnetic susceptibility for $\xi \geq 1$ are shown

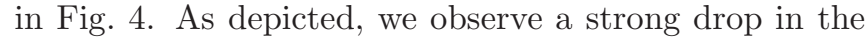
magnetic susceptibility above the isotropic point, i.e., in the regime $\xi_{c 2} \gtrsim 1.1$. From here, no ordering instability is found in the RG flow and the susceptibility rapidly looses the $120^{\circ}$ Néel order signature. While the $120^{\circ}$ Néel order peaks die out quickly, AFM stripe fluctuation signatures emerge (at points of the Brillouin zone according to Fig. 1). The transition appears to be of first order according to a pronounced kink in the maximal susceptibility upon varying $\xi$. While we do not find a breakdown of the flow that would indicate magnetic ordering, we still obtain strong collinear AFM stripe fluctuations (in agreement with [26]) signalled by an unstable RG flow that develops oscillations sensitively depending on the frequency discretization. These fluctuation tendencies are also seen at higher $\xi$ where the peak structure is still visible along the $k_{x}$-direction. However, these peaks are strongly broadened along the $k_{y}$-axis, i.e., smeared between the two c-AFM ordering vector positions. This indicates a fast exponential decay of spin correlations be- tween the $J^{\prime}$-chains. There are proposals in the literature that the (supposedly) disordered regime splits into two (gapped or gapless) different spin liquid phases [25]. In principle this would correspond to a change of diffuse spectral weight in the magnetic susceptibility going from an ungapped to a gapped system. We do not find clear indication for this scenario. At large $\xi$ the susceptibility does not change significantly with increasing $\xi$, which we studied by computing scenarios up to $\xi=5$ in steps of 0.2 in $\xi$.

In conclusion, we have used the pseudo-fermion functional renormalization group to study the different phases of the anisotropic triangular lattice. We find that upon variation of the anisotropy parameter $\xi$, the system divides into a Néel order, spiral order, and a disordered phase with c-AFM stripe fluctuations. We find evidence for a second order transition between the first two and a first order transition between the last two of these phases. We are confident that our method is a suitable starting point to discuss various other problems in the field of frustrated magnetism.

We thank P. Schmitteckert for his numerical support. We thank D. A. Abanin, B. A. Bernevig, A. Läuchli, K. P. Schmidt, S. Sondhi, O. Tchernyshyov, S. Wessel, and, in particular, P. Wölfle for discussions. JR is supported by DFG-FOR 960. RT is supported by a Feodor Lynen Fellowship of the Humboldt Foundation and Alfred P. Sloan Foundation funds. 
[1] G. Misguich and C. Lhuillier, in Frustrated Spin Systems (World Scientific, Singapore, 2004).

[2] A. W. Sandvik and J. Kurkijärvi, Phys. Rev. B 43, 5950 (1991).

[3] S. R. White, Phys. Rev. Lett. 69, 2863 (1992)

[4] U. Schollwöck, Rev. Mod. Phys. 77, 259 (2005).

[5] D. J. J. Farnell and R. F. Bishop, in Quantum Magnetism, Vol. 645 of Lecture Notes in Physics (SpringerVerlag, Berlin, 2004).

[6] L. Capriotti, A. E. Trumper, and S. Sorella, Phys. Rev. Lett. 82, 3899 (1999).

[7] D. Poilblanc, M. Mambrini, and D. Schwandt, arXiv:0912.0724.

[8] F. Wegner, Ann. Phys. (Leipzig) 3, 77 (1994)

[9] F. Mila and K. P. Schmidt, arXiv:1005.2495.

[10] F. Verstraete, J. I. Cirac, and V. Murg, Adv. Phys. 57, 143 (2008).

[11] G. Evenbly and G. Vidal, Phys. Rev. Lett. 102, 180406 (2009)

[12] G. Evenbly and G. Vidal, arXiv:0904.3383.

[13] J. Reuther and P. Wölfle, Phys. Rev. B 81, 144410 (2010).

[14] R. Coldea, D. A. Tennant, A. M. Tsvelik, and Z. Tylczynski, Phys. Rev. Lett. 86, 1335 (2001).

[15] Y. Shimizu, K. Miyagawa, K. Kanoda, M. Maesato, and G. Saito, Phys. Rev. Lett. 91, 107001 (2003).

[16] T. Itou, A. Oyamada, S. Maegawa, M. Tamura, and R. Kato, Phys. Rev. B 77, 104413 (2008).

[17] D. Heidarian, S. Sorella, and F. Becca, Phys. Rev. B 80, 012404 (2009).

[18] K. Kohno, Phys. Rev. Lett. 103, 197203 (2009).

[19] M. Q. Weng, D. N. Sheng, Z. Y. Weng, and R. J. Bursill, Phys. Rev. B 74, 012407 (2006).

[20] J. Merino, R. H. McKenzie, J. B. Marston, and C. H. Chung, J. Phys.: Condens. Matter 11, 2965 (1999).

[21] S.-Q. Shen and F. C. Zhang, Phys. Rev. B 66, 172407 (2002).

[22] R. F. Bishop, P. H. Y. Li, D. J. J. Farnell, and C. E. Campbell, Phys. Rev. B 79, 174405 (2009).

[23] T. Pardini and R. R. P. Singh, Phys. Rev. B 77, 214433 (2008).

[24] Z. Weihong, R. H. McKenzie, and R. R. P. Singh, Phys. Rev. B 59, 14367 (1999).

[25] S. Yunoki and S. Sorella, Phys. Rev. B 74, 014408 (2006).

[26] O. A. Starykh and L. Balents, Phys. Rev. Lett. 98, 077205 (2007).

[27] C. Wetterich, Phys. Lett. B 301, 90 (1993).

[28] T. R. Morris, Int. J. Mod. Phys. A 9, 2411 (1994).

[29] C. Honerkamp, M. Salmhofer, N. Furukawa, and T. M. Rice, Phys. Rev. B 63, 035109 (2001).

[30] R. Hedden, V. Meden, T. Pruschke, and K. Schönhammer, J. Phys.: Condes. Matter 16, 5279 (2004).

[31] A. A. Katanin, Phys. Rev. B 70, 115109 (2004).

[32] M. Salmhofer, C. Honerkamp, W. Metzner, and O. Lauscher, Prog. Theor. Phys. 112, 943 (2004).

[33] I. Affleck and J. B. Marston, Phys. Rev. B 37, 3774 (1988).

[34] J. B. Marston and I. Affleck, Phys. Rev. B 39, 11538 (1989).

[35] P. W. Anderson, Phys. Rev. 86, 694 (1952).
[36] J. Brinckmann and P. Wölfle, Phys. Rev. B 70, 174445 (2004).

\section{Supplementary material}

Néel order to spiral order transition. In the following we have resolved the Néel order to spiral order transition in higher resolution varying the anisotropy parameter $\xi$ in small stepwidths (see Fig. 5). We find that the previous Néel peak first broadens along $k_{x}$ and then smoothly splits up into two peaks which then evolve along the Brillouin-zone boundary. This process comes along with increasing spectral weight at the corners of the Brillouin zone. Note that as a consequence of the periodicity in momentum space, the emerging peak structure at $k_{y}=0$ presents the tails of the broadened Néel peak. Increasing $\xi$ further towards the isotropic triangular point (see Fig. (3) the split peaks move towards the corner position until at $\xi=1$ the hexagonal symmetry of the susceptibility is reached. As the susceptibility evolves completely smoothly through the transition, we find it to be of second order. We identify the wave vector of the corresponding long range-ordered phases with the position of the maximal susceptibility. From this we locate the transition point at such $\xi$ where the peaks split and above which the order becomes incommensurate. From Fig. 5 a transition at $\xi \approx 0.61$ can be read off. In the calculations for this figure we kept correlations up to a length of 5 lattice spacings (as compared to 7 lattice spacings in Fig. (3)and Fig. (4). In addition, the number of discrete frequencies has been slightly reduced in Fig. 5 . These reductions generally result in lower and broader peaks, which demonstrates that (according to the fact that the phases in this parameter regime are long range-ordered) there are still finite size effects in the susceptibility. Especially at the isotropic triangular point where additional lattice symmetries enable us to treat much longer correlations, we are able to resolve peaks at bigger system sizes which, in units of Fig. (4 reach heights up to 12 in $1 / J$, while the structure of the susceptibility is unchanged. However, in order to be able to detect long range order, for which the breakdown of the smooth RG-flow is the relevant criterion, we found it sufficient to use 5 or 7 lattice spacings as the maximal length of correlations. Another effect seen in a finite size scaling is that with increasing length of correlations (and also increasing number of discrete frequencies), the transition point moves towards slightly higher $\xi$. Therefore from a finite size scaling we estimate the transition to be approximately centered between $\xi=0.6$ and $\xi=0.7$.

High anisotropy regime. We provide more detailed data on the strong anisotropy regime (Fig. 6). As found there, the strong decay of correlations between the effectively weakly coupled one-dimensional chains is already dominant, which manifests itself in a strong smearing of the magnetic susceptibility along $k_{y}$. As $\xi$ is increased, the spectral weight of the susceptibility gets more and more reduced, while the fluctuation structure remains 

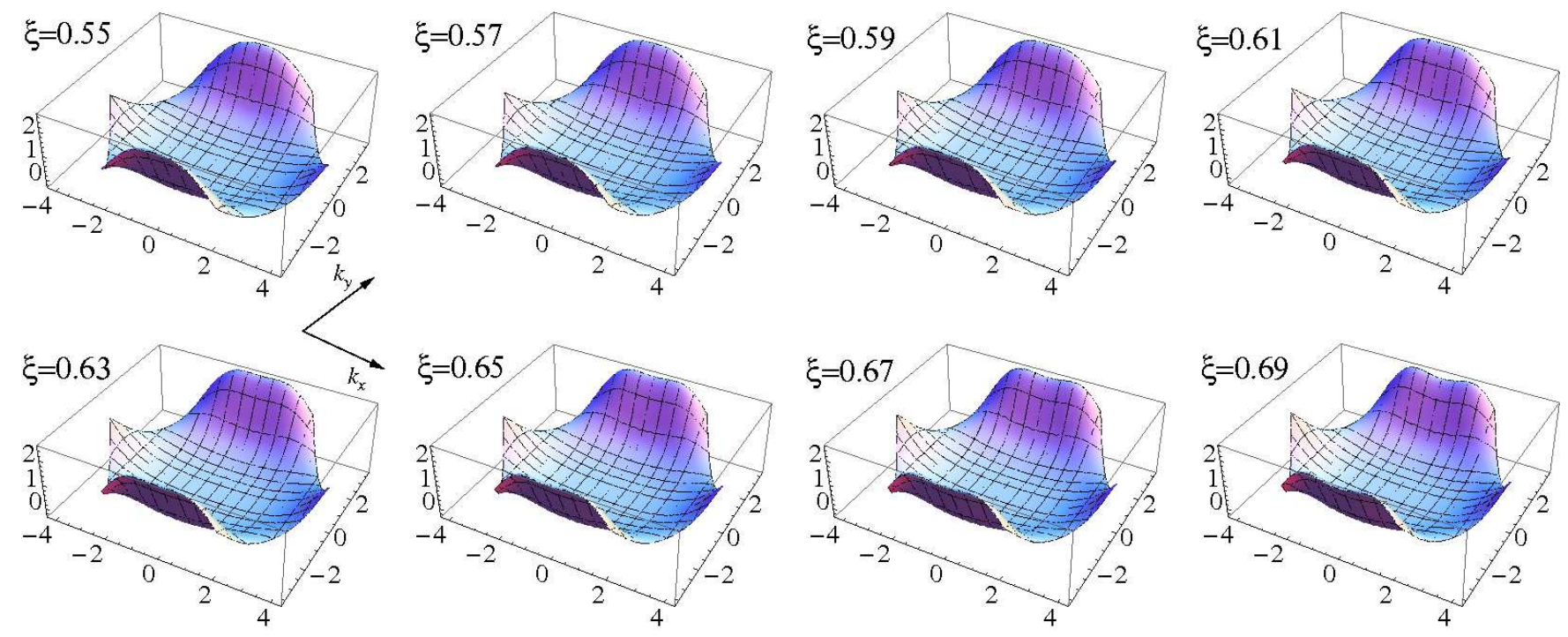

FIG. 5. Static magnetic susceptibility, varying $\xi$ from the AFM Néel side across the phase transition to spiral ordering. The already broadened AFM Néel peak splits up into two incommensurate peaks that successively shift towards the edges of the Brillouin zone where the $120^{\circ}$ Néel order peaks reside.
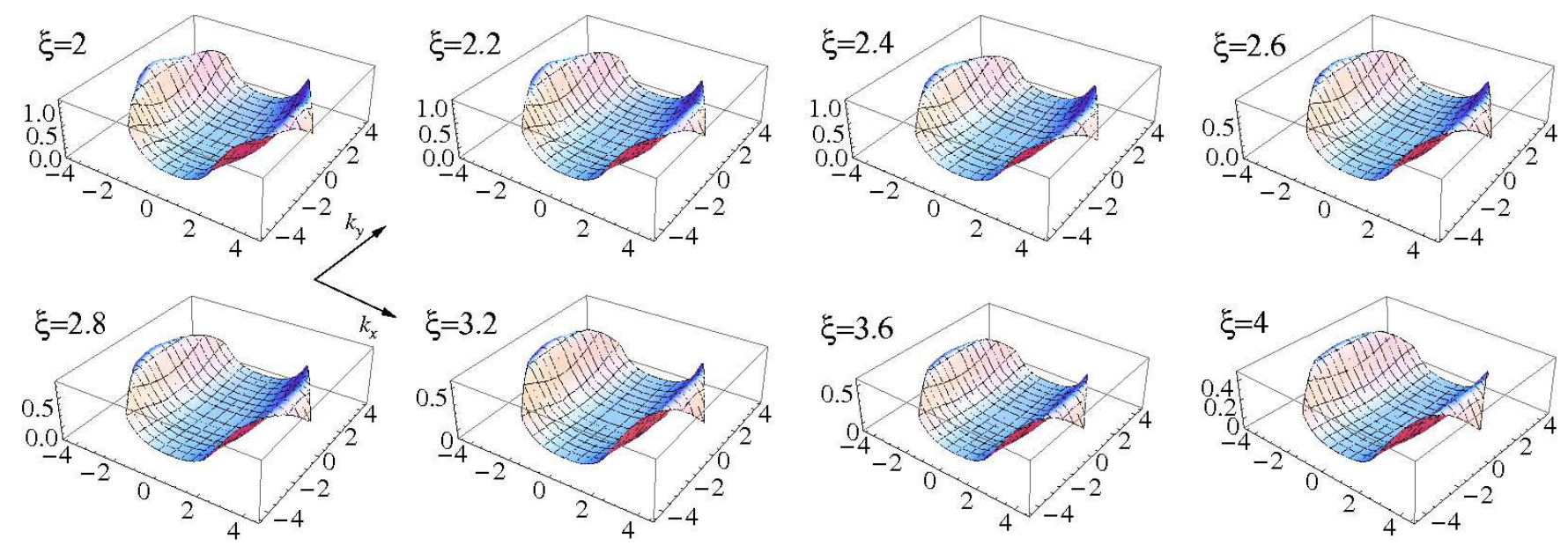

FIG. 6. Static magnetic susceptibility, varying $\xi$ in the strongly anisotropic regime from $\xi=2$ to $\xi=4$. While the spectral structure remains rather invariant of C-AFM type which is strongly smeared along the $k_{y}$ direction, the total spectral weight slightly decreases with increasing $\xi$.

rather stable. We only observe reminiscences of C-AFM fluctuations smeared along $k_{y}$. From there, we do not find clear indication for a bi-spin liquid scenario suggested in the literature for that regime, where one magnetically disordered phase should be gapped while the other should be gapless [Yunoki and Sorella, Phys. Rev. B 74, 014408 (2006).]. A transition between those phases should manifest itself in the susceptibility by some jump in the spectral weight. In general, at large anisotropies, energy scales involved in the coupling between the chains become so small that a caveat has to be given from the frequency discretization incorporated in the numerics, which sets a lower bound of energy scales to be resolved by our method. We varied the frequency mesh in the lower energy regime, and found no notable variation of our results. While this should hold for the anisotropy regime shown in the manuscript, we cannot exclude different physical scenarios for even higher anisotropy. 